(1968) .

${ }^{5}$ R. Beck, H. J. Mang, and P. Ring, Z. Phys. 231, 26 (1970).

${ }^{6} \mathrm{R}$. R. Hilton, in Proceedings of the Symposium on Present Status and Novel Developments in the Nuclear Many-Body Problem, Rome, September 1972, edited by F. Calogero and C. Ciofi degli Atti (Editrice Compositori, Bologna, 1973), Vol. 1, p. 669.

${ }^{7} \mathrm{R}$. R. Hilton, in Proceedings of the Extended Nuclear Physics Seminar on Heavy-Ion, High-Spin States and Nuclear Structure, Trieste, September 1973 (International Atomic Energy Agency, Vienna, 1975), Vol. II, p. 227, IAEA-SMR. 14/23.

${ }^{8}$ R. R. Hilton, in Proceedings of the International Symposium on Highly Excited States in Nuclei, Jülich, Federal Republic of Germany, 1975, edited by A. Faessler, C. Mayer-Böricke, and P. Türek (Kernforschung- sanlage Jülich GmbH, Jülich, Federal Republic of Germany, 1975), Vol. 1, p. 33.

${ }^{9}$ I. Hamamoto, Nucl. Phys. A271, 15 (1976).

${ }^{10}$ E. R. Marshalek and A. L. Goodman, Nucl. Phys. A294, 92 (1978) .

${ }^{11}$ F. Grümmer, K. W. Schmid, and A. Faessler, Nucl. Phys. A308, 77 (1978) .

${ }^{12}$ A. L. Goodman, Phys. Rev. Lett. 42,357 (1979).

${ }^{13} \mathrm{H}$. J. Mang and R. R. Hilton, in Proceedings of the Joint Nuclear Physics Spring Meeting of Deutsche Physikalische Gesellschaft, Nederlandse Natuurkundige Vereniging, Dansk Fysisk Selskab, and Belgische Natuurkundige Vereniging-Société Belge de Physique, Gent, Belgium, March 1979 (unpublished).

${ }^{14}$ S. Cohen, F. Plasil, and W. J. Swiatecki, Ann. Phys. (N.Y.) 82, 557 (1974).

${ }^{15}$ A. Bohr and B. Mottelson, Phys. Scr。10A, 13 (1974).

\title{
Delta-Electron Emission in Deep-Inelastic Heavy-Ion Collisions
}

\author{
Gerhard Soff \\ Gesellschaft für Schwerionenforschung, 6100 Darmstadt, Germany
}

and

Joachim Reinhardt, Berndt Müller, and Walter Greiner

Institut für Theoretische Physik der Johann Wolfgang Goethe-Universität, 6000 Frankfurt am Main, Germany (Received 21 September 1979)

This paper reports calculations of the influence of a reaction time $T>10^{-21} \mathrm{~s}$ in deepinelastic $\mathrm{Xe}-\mathrm{Pb}$ collisions on the energy spectrum of $\delta$ electrons ejected in the same collision. It is shown that the lifetime of the superheavy composite system causes pronounced oscillations of width $\epsilon=h / T$ in the electron distribution, which survive the inclusion of multistep excitations and the folding with a lifetime distribution function. This effect may serve as an atomic clock for deep-inelastic collisions.

Deep-inelastic heavy-ion scattering ${ }^{1}$ has become one of the best-studied phenomena in nuclear heavy-ion physics. To explain a wide range of observations two kinds of models have been proposed: microscopic models (shell-model fragmentation theory, ${ }^{2}$ time-dependent Hartree-Fock, ${ }^{3}$ etc.) and statistical models (diffusion theory, ${ }^{4}$ etc.). In the diffusion model, the experimentally observed widths of the fragment mass, charge, angular momentum, and energy distributions are in first approximation proportional to the available reaction time $T$. The magnitude of this time, however, must usually be determined by semiempirical procedures, ${ }^{1,4,5}$ which yield times in the range $10^{-21} \mathrm{~s} \lesssim T \lesssim 10^{-20} \mathrm{~s}$. As the basic assumptions of the various models are not acessible to direct experimental verification, it is extremely difficult to discriminate between the various proposed models. It would, therefore, be of great interest to have an independent clock for the time scale in deep-inelastic nuclear reactions. In the following, we propose a measurement of $T$ by means of the kinetic energy distribution of ejected $\delta$ electrons. Recent experiments ${ }^{6-8}$ have confirmed earlier predictions ${ }^{9}$ that inner-shell ionization is highly sensitive to the short-time structure of the electric field generated by the two nuclei during a heavy-ion collision.

Accordingly, the $\delta$-electron distribution may be employed ${ }^{10}$ for the analysis of electronic binding energies and the high momentum components of bound-state wave functions in the transient united atom. ${ }^{11}$ The spectrum has an exponential shape, the steepness being a function of the combined nuclear charge $Z=Z_{1}+Z_{2}$ and the minimal distance of approach of the two nuclei. For a typical center-of-mass energy of $3 \mathrm{MeV} / \mathrm{u}$, a minimal distance of $15 \mathrm{fm}$ corresponds to a time scale of 
the order of $10^{-21} \mathrm{~s}$. Any modification of this time structure due to nuclear reactions will produce observable changes in the ionization process.

The simplest possible modification is a nuclear sticking time $T$ that separates the incoming and outgoing Coulomb trajectory. It is easy to see that this corresponds to a phase shift between the ionization amplitudes from the two parts of the Coulomb orbit, the magnitude of which depends on the energy transferred to the electron.

The utilization of atomic ionization measurements for the determination of nuclear reaction times was first suggested by Ciocchetti, Molinari, and Malvano. ${ }^{12}$ Blair et al. ${ }^{13}$ recently succeeded in measuring the ionization amplitude for the half trajectory by investigating a proton resonance in ${ }^{58} \mathrm{Ni}$. The influence of a time delay on the bremsstrahlung spectrum was discussed by Eisberg, Yennie, and Wilkinson, ${ }^{14}$ the influence on quasimolecular spectra and $K \times$ rays by Bromley ${ }^{15}$ and two of the authors and recently by Anholt, ${ }^{16}$ Röhl, Hoppenau, and Dost and Chemin et al. ${ }^{17}$ Rafelski, Müller, and Greiner ${ }^{18}$ proposed that a nuclear delay time could increase spontaneous positron production in heavy-ion collisions. In this Letter we show that there is a pronounced effect on the electron spectrum.

We now turn to the theoretical concepts for inner-shell ionization. We have to solve the timedependent Schrödinger equation $i \hbar \partial \dot{\psi}_{i} / \partial t=H(R(t)) \psi_{i}$, with the relativistic Dirac Hamiltonian $H$. Since binding energies and wave functions change strongly as function of the internuclear separation $R$, the total wave function $\psi_{i}$ is expanded in terms of the adiabatic basis states $\varphi_{j}(R(t))$ which are solutions of the stationary two-center Dirac equation $H \varphi_{j}=E_{j}(R) \varphi_{j}$. We then obtain a set of coupled differential equations for the occupation amplitudes $a_{i j}$ of state $\varphi_{j}$ by electron number $i$ :

$$
\begin{aligned}
\dot{a}_{i j}(t)=-\boldsymbol{S}_{k} a_{i k}(t) & \left\langle\varphi_{j}|(\partial / \partial t)| \varphi_{k}\right\rangle \\
& \times \exp \left\{-(i / \hbar)\left(\chi_{k}-\chi_{j}\right)\right\} .
\end{aligned}
$$

The adiabatic electron energy enters via the phase

$$
\chi_{j}(t)=\int^{t} d t^{\prime} E_{j}\left(R\left(t^{\prime}\right)\right) .
$$

The mechanism by which a nuclear time delay influences the excitation amplitude becomes most transparent in first-order perturbation theory, where Eq. (1) is solved explicitly by

$$
\begin{aligned}
a_{i j}(t)=- & \int_{-\infty}^{t} d t^{\prime}\left\langle\varphi_{j}\left|\left(\partial / \partial t^{\prime}\right)\right| \varphi_{i}\right\rangle \\
& \times \exp \left\{(i / \hbar)\left[\chi_{j}\left(t^{\prime}\right)-\chi_{i}\left(t^{\prime}\right)\right]\right\} .
\end{aligned}
$$

This perturbative treatment correctly describes ${ }^{19}$ the ionization probability except for an overall normalization factor.

If we denote the ionization amplitude at the end of the incoming Coulomb orbit by $a_{i j}{ }^{\prime}=a_{i j}(t=0)$, we find that the probability of ionizing an electron from state $i$ into the continuum state $j$ for a fixed delay time $T$ is given by

$$
\begin{aligned}
& d P_{i}(\Delta E, T) / d E_{j} \\
& \quad=4\left|a_{i j}{ }^{\prime}\right|^{2} \sin ^{2}\left[(T / 2) \Delta E_{i j}+\arg \left(a_{i j}{ }^{\prime}\right)\right] .
\end{aligned}
$$

Here $\Delta E_{i j}=E_{j}-E_{i}$ is the energy difference between states $i$ and $j$ in the nuclear compound configuration. Obviously, $P_{i j}$ exhibits regular oscillations in the $\delta$-electron energy $E_{j}$ of width $\epsilon(T)$ $=h / T$. The minima go through zero in this ap-

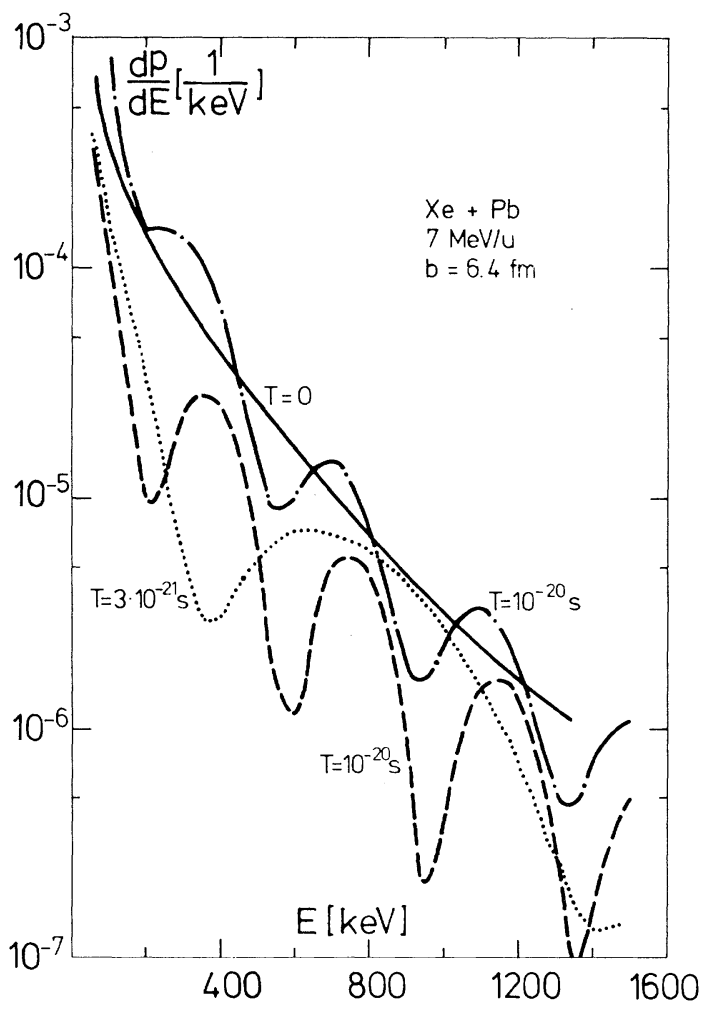

FIG. 1. Differential emission probability with respect to kinetic $\delta$-electron energy. A coincidence with created $1 s \sigma$ formation is required. The nuclear delay times $T=0,3 \times 10^{-21} \mathrm{~s}$, and $10^{-20} \mathrm{~s}$ are considered. The dash-dotted line represents the total $\delta$-electron spectrum $\left(T=10^{-20} \mathrm{~s}\right)$ stemming from $1 s \sigma, 2 s \sigma$, and $3 s \sigma$ ionization. 
proximation. These oscillations became slightly damped if higher-order and many-electron effects are included in the calculation. To show that much of the effect still survives we have solved Eq. (1) by coupled-channels calculation $\mathbf{s}^{20}$ for the system $\mathrm{Xe}-\mathrm{Pb}$ (or $\mathrm{Bi}$ ). This system is ideal both from the nuclear and the atomic physics sides (it is asymmetric enough to allow for a separation of the quasimolecular $1 s \sigma$ and $2 p_{1 / 2} O$ states) and has been widely studied experimentally. ${ }^{21}$

Assuming $7-\mathrm{MeV} / \mathrm{u}$ bombarding energy, grazing impact parameter $b=6.4 \mathrm{fm}$, and fully occupied $K, L$, and $M$ shells, we find the electron spectra shown in Fig. 1. Without nuclear reaction time the spectrum is a smoothly falling function (solid line, $T=0$ ), while a sticking time $T=10^{-20} \mathrm{~s}$ produces oscillations with a period of $400 \mathrm{keV}$ and a maximum-to-minimum ratio of ca. 3.5:1. This ratio is considerably increased (to $15: 1$ ) if one observes the lead $K$ vacancy in coincidence. In this way, one obtains a suppression of the contributions from all electrons except those originating form the $1 s \sigma$ level, i.e., the most deeply

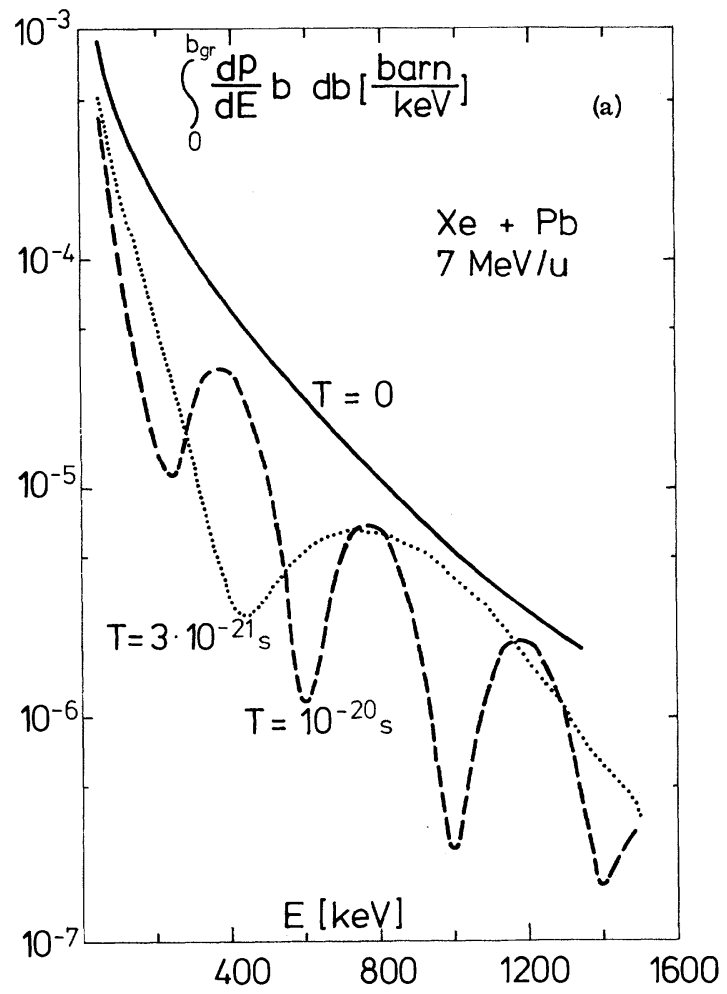

bound electronic state that is also most sensitive to nuclear details.

The range of nuclear delay times that lead to observable effects is limited below by the requirement that at least one oscillation must be observed.

The upper limit for $T$ is given by the experimental energy resolution and the variation of the electronic transition energy $\Delta E_{i j}$ during the nuclear reaction. If we take $\epsilon=10 \mathrm{keV}$ as the smallest detectable period, we obtain a time range $10^{-21} \mathrm{~s}<T<4 \times 10^{-19} \mathrm{~s}$ that is accessible to observation.

In the following we discuss the effects that could conceivably destroy the oscillatory pattern as seen in Fig. 1: (1) It is impossible to select a unique impact parameter $b$ in deep-inelastic collisions. The main effect of variation of $b$ is a change in the phase of $a_{i j}{ }^{\prime}$ in Eq. (4) and hence of the position of the minima in $d P / d E$. Figure 2(a) shows that the oscillations still prevail, even if we integrate over all impact parameters $b \leqslant b$ grazing that lead to a nuclear reaction. The $\max / \min$ ratio is virtually unaltered; only the

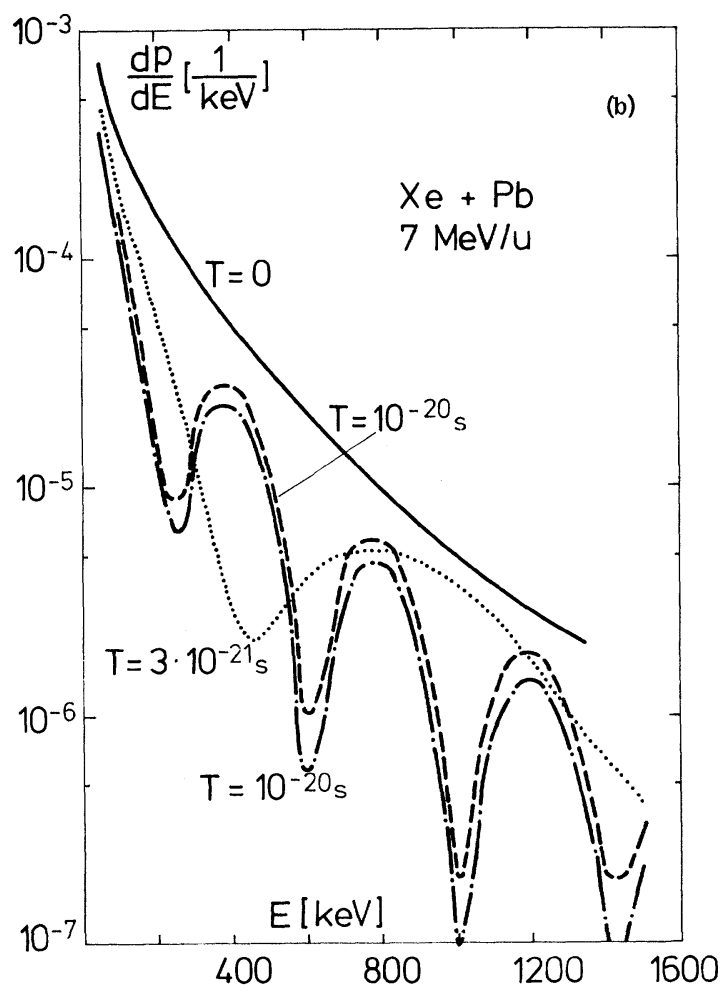

FIG. 2. (a) Notation as in Fig. 1. Integration over all impact parameters $b \leqslant b_{\text {grazing }}$ that lead to a nuclear reaction. (b) The same as in Fig. 1 for $\delta=3 \mathrm{fm}$. In addition, the dash-dotted line represents the $\delta$-electron distribution under the assumption of a relative laboratory energy loss of $10 \%$ on the outgoing path of the trajectory. 
positions are slightly shifted. (2) Energy dissipation destroys the symmetry between the incoming and outgoing branches of the Coulomb trajectory. We show the effect of this modification in Fig. 2(b), where was assumed a relative laboratory energy loss of $10 \%$, corresponding to 100 $\mathrm{MeV}$. We find that the spectrum as a whole is lowered, but the oscillations remain qualitatively unchanged. We have also calculated the influence of these effects on the total spectrum and find the same results. (3) Dissipation of angular momen- tum in the nuclear reaction cannot change the spectrum. This has been confirmed by separate calculations. (4) For any chosen set of experimental parameters, the nuclear reaction time $T$ may (and will) not be sharp but distributed over a certain range. The influence of a distribution function $f(T) \neq \delta\left(T-T_{0}\right)$ can be most illustratively seen in perturbation theory. If we take a Gaussian centered at $T_{0}$,

$$
f(T)=\tau^{-1}(2 \pi)^{-1 / 2} \exp \left[-\left(T-T_{0}\right)^{2} / 2 \tau^{2}\right]
$$

and $\tau \lesssim 2 T_{0}$, Eq. (4) is modified to

$$
d p / d E=2\left|a_{i j}{ }^{\prime}\right|^{2}\left\{1-\exp \left[-\left(\tau \Delta E_{i j}\right)^{2} / 2\right] \cos \left[T_{0} \Delta E_{i j}+2 \arg \left(a_{i j}{ }^{\prime}\right)\right]\right\} .
$$

For increasing electron energy, the oscillations become more and more damped. Remembering that the $n$th oscillation occurs at $\Delta E_{i j}=2 \pi n / T_{0}$, the number of observable oscillations is limited to $n<T_{0} / \pi \tau$. The ratio $T_{0} / \tau$, accordingly, limits the range of detectable delay times. It also indicates that the reaction time must be fixed in the experiment as well as possible. If we take $f(T)$ $=(1 / \tau) \exp (-T / \tau)$, the shape of the spectrum is slightly altered but all oscillations vanish, because $T=0$ is the most probable delay time.

We conclude that we have demonstrated the existence of a precise clock which may serve to measure absolute times involved in deeply inelastic encounters and other processes.

This work was supported in part by the Bundesministerium für Forschung und Technologie.

\footnotetext{
${ }^{1}$ See the review by W. U. Schröder and J. R. Huizenga, Ann. Rev. Nucl. Sci. 27, 465 (1977), and further references therein.

${ }^{2}$ J. A. Maruhn, J. Hahn, H. J. Lustig, K. H. Ziegenhain, and W. Greiner, in Proceedings of the Erice School on Heavy-Ion Interactions at High Energy, Erice, 1979, to be published.

${ }^{3}$ R. Y. Cusson, R. K. Smith, and J. A. Maruhn, Phys. Rev. Lett. 36, 1166 (1976).

${ }^{4}$ C. Riedel, G. Wolschin, and W. Nörenberg, Z. Phys. A 290,47 (1979).

${ }^{5}$ K. E. Rehm, H. Essel, K. Hartel, P. Kienle, H. J. Körner, P. Sperr, and W. Wagner, Phys. Lett. 86B, 256 (1979).

${ }^{6}$ J. S. Greenberg, H. Bokemeyer, H. Emling, E. Grosse, D. Schwalm, and F. Bosch, Phys. Rev. Lett. 39, 1404 (1977).

${ }^{7}$ R. Anholt and W. E. Meyerhof, Phys. Lett. $64 \mathrm{~A}, 381$ (1978).
}

${ }^{8}$ D. Liesen, P. Armbruster, H. H. Behnke, and S. Hagmann, Z. Phys. A 288, 417 (1978).

${ }^{9}$ W. Betz, G. Soff, B. Müller, and W. Greiner, Phys. Rev. Lett. 37, 1046 (1976); G. Soff, W. Betz, B. Müller, W. Greiner, and E. Merzbacher, Phys. Lett. 65A, 19 (1978).

${ }^{10} \mathrm{G}$. Soff, B. Müller, and W. Greiner, Phys. Rev. Lett. $\underline{40}, 540$ (1978).

${ }^{11}$ C. Ko zhuharov, P. Kienle, D. H. Jakubassa, and M. Kleber, Phys. Rev. Lett. 39, 540 (1977); F. Bosch, H. Krimm, B. Martin, B. Povh, Th. Walcher, and K. Traxel, Phys. Lett. 78B, 568 (1978).

${ }^{12}$ G. Ciocchetti, A. Molinari, and R. Malvano, Nuovo Cimento 29, 1262 (1963); G. Ciocchetti and A. Molinari, Nuovo Cim. 40B, 69 (1965).

${ }^{13}$ J. S. Blair, P. Dyer, K. A. Suover, and T. A. Trainor, Phys. Rev. Lett. 41,1712 (1978).

${ }^{14}$ R. M. Eisberg, D. R. Yennie, and D. H. Wilkinson, Nucl. Phys. 18, 338 (1960).

${ }^{15} \mathrm{D}$. A. Bromley, in Proceedings of the International Conference on Reactions between Complex Nuclei, Nashville, 1974, edited by R. L. Robinson, F. K. McGowan, J. B. Ball, and J. H. Hamilton (North Holland, Amsterdam, 1974), p. 603.

${ }^{16}$ R. Anholt, Z. Phys. A $\underline{292}, 123$ (1979), to be published.

${ }^{17}$ S. Röhl, S. Hoppenau, and M. Dost, Phys. Rev. Lett. 43,1300 (1979); J. F. Chemin, S. Andriamonje, J. Rotutier, J. P. Thibaud, S. Joly, and J. Uzureau, in Proceedings of the Eleventh International Conference on the Physics of Electronic and Atomic Collisions, Kyoto, 1979, to be published.

${ }^{18} \mathrm{~J}$. Rafelski, B. Müller, and W. Greiner, Z. Phys. A 285,49 (1979).

${ }^{19}$ G. Soff, W. Greiner, W. Betz, and B. Müller, Phys. Rev. A 20, 169 (1979).

${ }^{20} \mathrm{~J}$. Reinhardt, B. Müller, W. Greiner, and G. Soff, Phys. Rev. Lett. $\underline{43}, 1307$ (1979).

${ }^{21}$ W. U. Schröder, J. R. Birkelund, J. R. Huizenga, K. L. Wolf, J. P. Unik, and V. E. Viola, Phys. Rev. Lett. $\underline{36}, 514$ (1976). 